\title{
Comparison of leading and next-to-leading logarithmic electroweak corrections to Higgs production
}

\author{
Fabio Siringo \\ Dipartimento di Fisica e Astronomia dell' Università di Catania and INFN Sezione di Catania, \\ Via S.Sofia 64, I-95123 Catania, Italy.
}

(Dated: July 30, 2018)

\begin{abstract}
Using soft-collinear effective theory, the leading-log radiative electroweak corrections are written in a closed and analytical form for the hadronic cross section of Higgs production through vector boson fusion, $q q \rightarrow q q H$, one of the most promising channels for studying the Higgs boson at the LHC. The simple leading-log resummation is compared with a full next-to-leading-log calculation, and its accuracy is found to be of order $1 \%$ up to $10 \mathrm{TeV}$, i.e. better than the accuracy of PDFs. Corrections are found to be larger than predicted by one-loop fixed order approximations at LHC energies. The method provides a simple way of incorporating the electroweak corrections in software packages, improving the accuracy of simulations.

PACS numbers: 12.15.Lk, 12.38.Cy, 14.80.Bn
\end{abstract}

\section{INTRODUCTION}

The recent discovery of a new boson at the Large Hadron Collider (LHC) 1, 2] will lead to a detailed study of its properties in the next years, and will require a careful comparison of the experimental data with the results of precise calculations for the Higgs sector of the Standard Model (SM). Vector boson fusion (VBF), $q q \rightarrow q q H$, is the second largest channel for detecting the Higgs boson. It is a pure electroweak process and its study is very important for determining the couplings and for a deeper knowledge of the Higgs sector [3, 4]. However, at the 10 Tev energy scale of LHC, even the electroweak radiative corrections become important [5, [6] and should be included in computer simulations together with QCD corrections $7-14]$. With a partonic centerof-mass energy $\sqrt{s}$ of several $\mathrm{TeV}$ - more than an order of magnitude larger than the masses $M_{W, Z}$ of the gauge bosons - the radiative corrections contain large Sudakov logarithmic terms $\alpha^{n} L^{m}$ where $\alpha=\alpha_{1,2}$ are the weak coupling constants and the $\operatorname{logarithms} L=\log \left(s / M_{W, Z}^{2}\right)$ emerge from the two different energy scales. These terms dominate the perturbative expansion and may even require resummation when the fixed order perturbation expansion breaks down. However, for VBF the scattering amplitude is proportional to the vacuum expectation value (VEV), and standard resummation methods do not apply because the effective operator is not a gauge singlet. That makes VBF a special interesting process to deal with.

The Sudakov logarithms can be regarded as infrared logarithms since they diverge as $M_{W, Z} \rightarrow 0$, and by using an effective theory they can be converted to ultraviolet logarithms and summed by standard renormalization group techniques. Quite recently the soft-collinear effective theory (SCET) 15 18] has been shown to provide a simple way to obtain the sum of the series of leading-logs (LL) $\alpha^{n} L^{n+1}$, next-to-leading-logs (NLL) $\alpha^{n} L^{n}$, next-tonext-to-leading-logs (NNLL) $\alpha^{n} L^{n-1}$, etc. [19 22]. In the effective theory the single terms contributing to the scattering amplitude are multiplied by the general factor [21]

$$
\begin{aligned}
& \mathcal{U}\left(\mu_{l}, \mu_{h}\right)=\exp \left[D_{0}\left(\alpha\left(\mu_{l}\right)\right)+D_{1}\left(\alpha\left(\mu_{l}\right)\right) \log \frac{\mu_{h}^{2}}{\mu_{l}^{2}}\right] \\
& \quad \times \exp \left\{-\int_{\mu_{l}}^{\mu_{h}} \frac{\mathrm{d} \mu}{\mu}\left[A(\alpha(\mu)) \log \frac{\mu^{2}}{\mu_{h}^{2}}+B(\alpha(\mu))\right]\right\} \\
& \quad \times \exp F\left(\alpha\left(\mu_{h}\right)\right)
\end{aligned}
$$

where $\mu_{l} \approx M_{Z}$ is the low energy scale and $\mu_{h} \approx \sqrt{s}$ is the high energy scale. The coefficient $F$ is the high scale matching coefficient, $D_{0}$ and $D_{1}$ are the low scale matching coefficients, $B$ is the non-cusp anomalous dimension and $A$ is the coefficient of the cusp anomalous dimension. The LL series is summed by the one-loop cusp anomalous dimension; the sum of the NLL series requires the two-loop cusp anomalous dimension, the one-loop noncusp anomalous dimension, and the one-loop low scale matching coefficient $D_{1}$; the NNLL series is given by the the three-loop cusp, two-loop $B$ and $D_{1}$, one-loop $D_{0}$ and $F$, etc. In fact the exponentiated form of Eq.(1) only requires the inclusion of electroweak corrections at low orders, while the unexponentiated form of fixed-order calculations would require electroweak corrections of any order for achieving the same accuracy. In some recent papers [23, 24] the method as been used for calculating the electroweak corrections to Higgs production via VBF. Numerical results at NLL order were obtained for the cross section, including the effect of parton distribution functions (PDFs) [24]. Most of the corrections were obtained in analytical form by SCET and might be easily included in the other software packages that have been developed, without the need of tedious loop calculations.

While PDFs imply a $3 \%$ error, many of the retained terms at NLL order are very small, less than $1 \%$, and could be neglected in order to speed up the numerical integration of the cross section. In this paper the simpler LL order resummation is compared with the full NLL calculation for Higgs production via VBF, and the accuracy of the two approximations is discussed. The LL calcu- 
lation of the cross section is found to deviate from the NLL result by less than $1 \%$ up to $10 \mathrm{TeV}$ center-of-mass energy. While the present study gives a useful evaluation of the accuracy in the SCET resummation, it provides a very simple and fast analytical way for including the electroweak radiative corrections in software packages.

In fact at NLL order the one-loop anomalous dimension turns out to be a $10 \times 10$ matrix in the operator basis [24], and the running integral in Eq.(1) requires a numerical computation. Moreover two-loop beta functions are required for the running of the couplings at the same order, yielding coupled equations that again must be solved by a numerical routine. On the other hand, at LL order the only one-loop term that is required is the cusp anomalous dimension, and by use of the simple uncoupled one-loop beta functions the running integral in Eq.(1) is analytical, yielding a diagonal correction factor for the differential cross section. Moreover, an exact cancellation of terms yields the same correction factor that would be found for the quark scattering $q q \rightarrow q q$, plus Higgs rescattering and wave function renormalization terms. NLL terms are still shown to be relevant for a full description of the dependence on scattering angles of the differential cross section, but such small dependence is averaged in the integrated cross section.

The paper is organized as follows: in Sec. II the kinematics of the VBF process is described and some details on the integration of the cross section are reported; the general calculation framework is described in Sec. III where the operator basis set is defined for the effective theory and matched onto the low scale and high scale gauge theories; in Sec. IV the running of the Wilson coefficients is discussed and the anomalous dimension is shown to take a simple diagonal form at LL order; explicit analytical expressions are derived for the running integral in Sec. $[\mathrm{V}$ and the numerical results at LL order and NLL order ar compared with other fixed order perturbative calculations in Sec. VI

\section{KINEMATICS OF VBF AND CROSS SECTION}

At tree-level, the Feynman diagrams for the process $q q \rightarrow q q H$ are shown in Fig.1. The two outgoing jets have a large rapidity gap that characterizes the VBF channel, and the background is usually suppressed by cuts (for

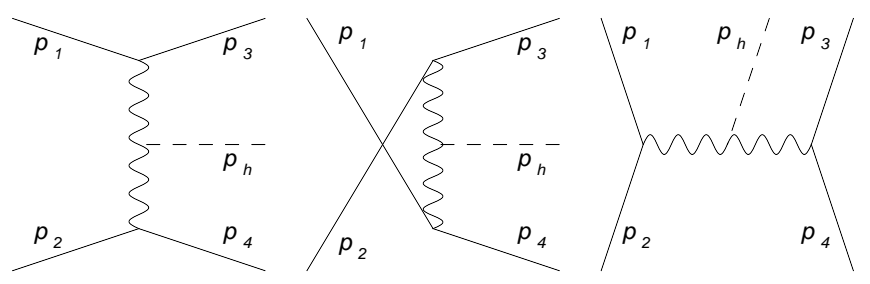

FIG. 1: Diagrams for electroweak Higgs production at treelevel: $t-, u-$ and $s$-channel diagrams are reported.
VBF cuts see e.g. Refs. [8, 25 27]).

We denote by $p_{1}, p_{2}$ the momenta of the incoming quarks, and by $p_{3}, p_{4}$ the momenta of the outgoing quarks (jets). The momentum of the Higgs boson is denoted by $p_{h}$, and it is assumed to be on-shell, $p_{h}^{2}=M_{h}^{2}$. Quark masses are neglected, $p_{1}^{2}=p_{2}^{2}=p_{3}^{2}=p_{4}^{2}=0$, and all momenta are taken to be incoming, according to the conventions of Ref. [23]. The kinematic can be expressed in terms of generalized Mandelstam variables:

$$
\begin{aligned}
s & =\left(p_{1}+p_{2}\right)^{2}, & t_{3}=\left(p_{1}+p_{3}\right)^{2}, & t_{4}=\left(p_{2}+p_{4}\right)^{2}, \\
u_{3} & =\left(p_{2}+p_{3}\right)^{2}, & u_{4}=\left(p_{1}+p_{4}\right)^{2} . &
\end{aligned}
$$

In SCET, a set of light-cone vectors is associated to each collinear direction. They are defined as $n_{i}=$ $\pm\left(1, \mathbf{n}_{i}\right), \bar{n}_{i}= \pm\left(1,-\mathbf{n}_{i}\right)$, with the plus (minus) sign for incoming (outgoing) particles. For the quarks $n_{i}=$ $\pm p_{i} / p_{i}^{0}$ while for the Higgs $\mathbf{n}_{h}=-\mathbf{p}_{h} / \sqrt{\left(p_{h}^{0}\right)^{2}-M_{h}^{2}}$.

The colliding quarks $(i=1,2)$ carry a fraction $x_{i}$ of the total hadron momenta,

$$
p_{i}^{\mu}=x_{i} E_{\mathrm{cm}} \frac{n_{i}^{\mu}}{2}, \quad n_{i}^{\mu}=(1,0,0, \pm 1)
$$

where $E_{\mathrm{cm}}$ is the center-of-mass energy, and the fractions $x_{i}$ are integrated over in the cross section by use of PDFs that describe their distribution in the proton. The momenta of the outgoing jets can be written as

$$
\begin{aligned}
& p_{3}=-E_{3}\left(1, \sin \theta_{3}, 0, \cos \theta_{3}\right), \\
& p_{4}=-E_{4}\left(1, \sin \theta_{4} \cos \varphi, \sin \theta_{4} \sin \varphi, \cos \theta_{4}\right),
\end{aligned}
$$

where $E_{i}>0$ are the energies and $\theta_{i}$ the angles with the axis of the beam, while $\varphi$ is the azimuthal angle between the outgoing quarks. The momentum of the Higgs is fixed by momentum conservation.

In the effective theory, in order to evaluate the cross section, we square the matrix elements of the effective operators, sum over helicities, flavors, channels and integrate over phase space:

$$
\begin{aligned}
\sigma_{\mathrm{EW}}= & \frac{1}{2 E_{\mathrm{cm}}^{2}} \int \frac{\mathrm{d} x_{1}}{x_{1}} \frac{\mathrm{d} x_{2}}{x_{2}} \mathrm{~d} \Phi_{3} \frac{1}{4} \sum_{\text {hel. flav. chan. }} \sum_{X, Y} \\
& \times \sum_{X} f_{i}\left(x_{1}\right) f_{j}\left(x_{2}\right) \hat{C}_{X}^{*} \hat{C}_{Y}\left\langle\hat{O}_{X}\right\rangle^{*}\left\langle\hat{O}_{Y}\right\rangle,
\end{aligned}
$$

where $\hat{O}_{X}$ are the effective operators and $f_{i}(x)$ are the PDF for the flavor $i$ at momentum fraction $x$, and a factor of $1 / 2$ must be included for identical particles in symmetric phase-space integrations. The Wilson coefficients $\hat{C}$ are obtained in terms of tree-level high scale coefficients $C$ by matching at the high scale, running down to the low scale and matching at the low scale according to Eq.(1),

$$
\hat{C}\left(\mu_{l}\right)=\mathcal{U}\left(\mu_{l}, \mu_{h}\right) \cdot C\left(\mu_{h}\right)
$$

which must be regarded as a matrix product in the oper- 
ator basis set. The three-body phase space $\mathrm{d} \Phi_{3}$ reads $[24]$

$$
\begin{aligned}
\int \mathrm{d} \Phi_{3}= & \int \prod_{i=3,4} \frac{\mathrm{d}^{3} p_{i}}{(2 \pi)^{3} 2 E_{i}} \frac{\mathrm{d}^{4} p_{h}}{(2 \pi)^{3}} \theta\left(-p_{h}^{0}\right) \delta\left(p_{h}^{2}-M_{h}^{2}\right) \\
& \times(2 \pi)^{4} \delta^{4}\left(p_{1}+p_{2}+p_{3}+p_{4}+p_{h}\right) \\
= & \frac{1}{2^{6} \pi^{4}} \int \prod_{i=3,4}\left[E_{i} \mathrm{~d} E_{i} \mathrm{~d}\left(\cos \theta_{i}\right)\right] \mathrm{d} \varphi \theta\left(\sum_{j=1}^{4} p_{j}^{0}\right) \\
& \times \delta\left[\left(\sum_{j=1}^{4} p_{j}\right)^{2}-M_{h}^{2}\right] \\
= & \frac{1}{2^{6} \pi^{4}} \int \mathrm{d}\left(\cos \theta_{3}\right) \mathrm{d}\left(\cos \theta_{4}\right) \mathrm{d} \varphi \mathrm{d} E_{3} \frac{E_{3} f_{1}}{f_{2}^{2}} .
\end{aligned}
$$

where

$f_{1}=x_{1} x_{2} E_{\mathrm{cm}}^{2}-M_{h}^{2}-\left[x_{1}+x_{2}+\left(x_{2}-x_{1}\right) \cos \theta_{3}\right] E_{\mathrm{cm}} E_{3}$

$f_{2}=\left[x_{1}+x_{2}+\left(x_{2}-x_{1}\right) \cos \theta_{4}\right] E_{\mathrm{cm}}-2\left[1-\cos \theta_{3} \cos \theta_{4}\right.$

$\left.-\cos \varphi \sin \theta_{3} \sin \theta_{4}\right] E_{3}$.

The remaining integrals in Eq. (7) must be carried out numerically with the boundary conditions

$$
\begin{aligned}
& 0 \leq \theta_{3}, \theta_{4} \leq \pi, \quad 0 \leq \varphi \leq 2 \pi, \\
& \frac{M_{h}^{2}}{E_{\mathrm{cm}}^{2}} \leq x_{1} \leq 1, \quad \frac{M_{h}^{2}}{x_{1} E_{\mathrm{cm}}^{2}} \leq x_{2} \leq 1, \\
& 0 \leq E_{3} \leq \frac{x_{1} x_{2} E_{\mathrm{cm}}^{2}-M_{h}^{2}}{\left[x_{1}+x_{2}+\left(x_{2}-x_{1}\right) \cos \theta_{3}\right] E_{\mathrm{cm}}}
\end{aligned}
$$

While we already included the bounds of the PDFs, the boundaries can be restricted by further cuts that we might impose on the phase space in the integration.

\section{EFFECTIVE THEORY AND OPERATOR BASIS SET}

Electroweak corrections can be obtained by SCET in the framework of Ref. [19, 22]. The extension to the VBF process was derived in Ref. 23, 24], and the explicit expressions are reported in Ref. [24]. Here we only consider the SM gauge group. Extensions like the minimal leftright symmetric gauge group [28] will be the subject of an other paper.

The first step consists of matching onto SCET at a high scale $\mu_{h} \sim \sqrt{s}$. Here the effects of symmetry breaking are suppressed, and the matching can be done in the unbroken gauge theory. Next we run the effective operators down to a low scale $\mu_{l} \sim M_{Z}$ by renormalization group equations. At the low-scale, the $W$ and $Z$ boson are integrated out: we match onto a $S U(3) \times U(1)$ effective theory, only containing gluons and photons. In this low-scale matching, the effects of $S U(2) \times U(1)$ symmetry breaking must be considered. They only enter in this low-energy matching.
At the high scale, the operators for VBF are given by [23, 24]

$$
\begin{aligned}
\mathcal{O}_{1 A, B} & =O_{1} O_{A}, O_{1} O_{B}, \\
\mathcal{O}_{2 A, B, C} & =O_{2}^{a} O_{A}^{a}, O_{2}^{a} O_{B}^{a}, O_{2}^{a} O_{C}^{a}, \\
\mathcal{O}_{3 A, B} & =O_{3} O_{A}, O_{3} O_{B}, \\
\mathcal{O}_{4 A, B, C} & =O_{4}^{a} O_{A}^{a}, O_{4}^{a} O_{B}^{a}, O_{4}^{a} O_{C}^{a} .
\end{aligned}
$$

In the Higgs sector we introduce the operators $O_{1}, \ldots, O_{4}$

$$
\begin{aligned}
O_{1} & =\Phi_{h}^{\dagger} \phi_{0}+\phi_{0}^{\dagger} \Phi_{h}, \\
O_{2}^{a} & =\Phi_{h}^{\dagger} T^{a} \phi_{0}-\phi_{0}^{\dagger} T^{a} \Phi_{h}, \\
O_{3} & =\Phi_{h}^{\dagger} \phi_{0}-\phi_{0}^{\dagger} \Phi_{h}, \\
O_{4}^{a} & =\Phi_{h}^{\dagger} T^{a} \phi_{0}+\phi_{0}^{\dagger} T^{a} \Phi_{h},
\end{aligned}
$$

where $\Phi_{h}=W_{n_{h}}^{\dagger} \phi_{n_{h}}$ is the collinear scalar doublet $\phi_{n_{h}}$ that gives rise to a Higgs boson, with its collinear Wilson line $W_{n_{h}}$. The field $\phi_{0}$ is a soft scalar that gives rise to a $\mathrm{VEV}$ when the symmetry is broken. For the quarks we introduce the operators

$$
\begin{aligned}
O_{A} & =\bar{\Psi}_{3} \gamma^{\mu} T^{a} \Psi_{1} \bar{\Psi}_{4} \gamma_{\mu} T^{a} \Psi_{2}, \\
O_{B} & =C_{F} \bar{\Psi}_{3} \gamma^{\mu} \Psi_{1} \bar{\Psi}_{4} \gamma_{\mu} \Psi_{2}, \\
O_{A}^{a} & =\bar{\Psi}_{3} \gamma^{\mu} T^{a} \Psi_{1} \bar{\Psi}_{4} \gamma_{\mu} \Psi_{2}, \\
O_{B}^{a} & =\bar{\Psi}_{3} \gamma^{\mu} \Psi_{1} \bar{\Psi}_{4} \gamma_{\mu} T^{a} \Psi_{2}, \\
O_{C}^{a} & =i \epsilon^{a b c} \bar{\Psi}_{3} \gamma^{\mu} T^{b} \Psi_{1} \bar{\Psi}_{4} \gamma_{\mu} T^{c} \Psi_{2},
\end{aligned}
$$

with the subscript $i=1, \ldots, 4$ that labels the momentum $p_{i}$ of the particle. Collinear Wilson lines $\Psi_{i}=W_{n_{i}}^{\dagger} \psi_{n_{i}}$ are included in all these fermion fields, according to collinear gauge invariance. In order to keep the notation as general as possible, the projectors $P_{R, L}=\left(1 \pm \gamma_{5}\right) / 2$ have been suppressed, and both left- and right-handed quarks have been allowed. Of course the field $\Psi_{i}$ is supposed to be a fermion doublet (singlet) if it is left-handed (righthanded).

Each helicity is considered separately and the contributions are combined at the end. It is quite obvious that the operators $\mathcal{O}_{A}$ and $\mathcal{O}_{C}^{a}$ can only make sense if the quarks are left-handed, while the operators $\mathcal{O}_{A}^{a}$ and $\mathcal{O}_{B}^{a}$ are allowed if at least one of the incoming particles is left-handed.

As discussed in Ref. 24] this operator basis is not complete, because it only suffices for quarks and for the $t$ channel. However for incoming anti-quarks and for the $u$-channel the corresponding operators are obtained by interchanging the particle labels. Thus we will only discuss the case of incoming quarks in the $t$-channel.

At LL and NLL order the tree-level high-scale matching suffices, and can be done in the unbroken phase of the electroweak gauge theory. The coupling of the scalar doublet to the gauge fields is described by the Lagrangian terms

$$
\begin{aligned}
\mathcal{L}= & \frac{1}{4}\left(g_{2}^{2} A_{\mu}^{a} A^{a \mu}+g_{1}^{2} B_{\mu} B^{\mu}\right) \Phi^{\dagger} \Phi \\
& +g_{1} g_{2} B^{\mu} A_{\mu}^{a} \Phi^{\dagger} T^{a} \Phi .
\end{aligned}
$$


In Fig. 1 we only need to consider the $t$-channel, because the $s$-channel contribution is suppressed in VBF. Matching onto the operators in Eq. (10), yields

$$
\begin{aligned}
& C_{1 A}(\mu)=\frac{i g_{2}^{4}(\mu)}{2 t_{3} t_{4}} \eta_{1} \eta_{2}, \quad C_{1 B}(\mu)=\frac{2 i g_{1}^{4}(\mu)}{3 t_{3} t_{4}} Y_{1} Y_{2}, \\
& C_{4 A}(\mu)=\frac{i g_{1}^{2}(\mu) g_{2}^{2}(\mu)}{t_{3} t_{4}} \eta_{1} Y_{2}, \\
& C_{4 B}(\mu)=\frac{i g_{1}^{2}(\mu) g_{2}^{2}(\mu)}{t_{3} t_{4}} Y_{1} \eta_{2} .
\end{aligned}
$$

while the other coefficients vanish at tree level. In order to keep the notation as general as possible, a new variable $\eta_{i}$ has been defined: $\eta_{i}=1$ if the particle with label $i$ is left-handed and $\eta_{i}=0$ if the particle is right-handed. The hypercharge is $Y=1 / 6$ for left-doublets, while for right-handed particles $Y=2 / 3$ for up-type quarks and $Y=-1 / 3$ for down-type quarks.

The running of the Wilson coefficients follows by the RG equation

$$
\mu \frac{\mathrm{d}}{\mathrm{d} \mu} C(\mu)=\gamma(\mu) C(\mu),
$$

in terms of the anomalous dimension $\gamma$ of the operators. That is a matrix equation, corresponding to the $10 \mathrm{op}-$ erators in Eq. (10). Using the notation of Eq. (1) the anomalous dimension can be written as the sum of cusp and non-cusp terms

$$
\gamma(\mu)=A(\alpha(\mu)) \log \frac{\mu^{2}}{\mu_{h}^{2}}+B(\alpha(\mu)),
$$

We only need the one-loop cusp anomalous dimension at LL order, while two-loop cusp and one-loop non-cusp terms were required at NLL order and were reported in detail in Ref. 24].

Finally, let us consider the low energy matching. The tree-level result suffices at LL order. The one-loop calculation was required at NLL order and was also reported in Ref. [24]. At low energies we must take into account the effects of electroweak symmetry breaking. We match onto a basis of operators in the broken phase of the gauge group.

For the Higgs part of the operator, given in Eq. (11), the soft scalar field simply attains a VEV and the collinear scalar field produces a Higgs. Thus at tree-level the low energy matching yields

$$
\left\{O_{1}, O_{2}^{a}, O_{3}, O_{4}^{a}\right\} \rightarrow\left\{1,0,0,-\delta^{a 3} / 2\right\} v h
$$

where $v$ is the VEV and $h$ is the Higgs field.

The quark part in Eq. (12) gets matched onto the set

$$
\begin{aligned}
& \hat{O}_{A}=\bar{u}_{3} \gamma^{\mu} u_{1} \bar{u}_{4} \gamma_{\mu} u_{2}, \\
& \hat{O}_{B}=\bar{u}_{3} \gamma^{\mu} u_{1} \bar{d}_{4} \gamma_{\mu} d_{2}, \\
& \hat{O}_{C}=\bar{d}_{3} \gamma^{\mu} d_{1} \bar{u}_{4} \gamma_{\mu} u_{2}, \\
& \hat{O}_{D}=\bar{d}_{3} \gamma^{\mu} d_{1} \bar{d}_{4} \gamma_{\mu} d_{2}, \\
& \hat{O}_{E}=\bar{d}_{3} \gamma^{\mu} u_{1} \bar{u}_{4} \gamma_{\mu} d_{2}, \\
& \hat{O}_{F}=\bar{u}_{3} \gamma^{\mu} d_{1} \bar{d}_{4} \gamma_{\mu} u_{2} .
\end{aligned}
$$

Here $u$ and $d$ denote up and down-type quarks. For operators $\hat{O}_{A}, \ldots, \hat{O}_{D}$, the pairs of fields $\bar{\psi} \gamma^{\mu} \psi$ can be lefthanded or right-handed, whereas in $\hat{O}_{E}$ and $\hat{O}_{F}$ all fields must be left handed. We match $\left\{\mathcal{O}_{1 A, B}, \mathcal{O}_{4 A, B, C}\right\}$ onto the operators $\left\{\hat{O}_{A}, \ldots, \hat{O}_{F}\right\}$ in Eq. (18), while we ignore $O_{2}^{a}$ and $O_{3}$ which vanish at tree level. The matching is described by the $6 \times 5$ matrix $R^{(0)}$ which is defined according to

$$
O_{i} \rightarrow v h \sum_{J} \hat{O}_{j} R_{j i}^{(0)}, \quad \hat{C}_{j}=v \sum_{i} R_{j i}^{(0)} C_{i},
$$

so that

$$
\sum_{i} C_{i} O_{i} \rightarrow \sum_{i} \hat{C}_{i} h \hat{O}_{i}
$$

The matrix $R^{(0)}$ follows

$$
R^{(0)}=\frac{1}{4}\left(\begin{array}{ccccc}
1 & 3 & -1 & -1 & 0 \\
-1 & 3 & -1 & 1 & 0 \\
-1 & 3 & 1 & -1 & 0 \\
1 & 3 & 1 & 1 & 0 \\
2 & 0 & 0 & 0 & -1 \\
2 & 0 & 0 & 0 & 1
\end{array}\right) .
$$

\section{ANOMALOUS DIMENSION AND RUNNING}

At LL order, one-loop corrections enter through the cusp anomalous dimension. For VBF the one-loop terms contributing to the anomalous dimension were derived in Ref. 23] and reported in detail in Ref. 24]. They can be written as the sum of three terms

$$
\gamma=\gamma_{C}+\gamma_{S}+\hat{\gamma}
$$

The first term $\gamma_{C}$ is the collinear anomalous dimension, it is diagonal and contains the large logarithms that contribute to the cusp anomalous dimension, plus wave function renormalization terms. Resummation at LL order requires the inclusion of these one-loop terms, and they are easily obtained by the sum of single-particle collinear terms [22, 29] for all the external particles

$$
\gamma_{C}=\sum_{i} \gamma_{C}^{i}+\gamma_{C}^{h}
$$

As reported in Ref. [24], with the shorthand

$$
L_{i}=\log \frac{\bar{n}_{i} \cdot p_{i}}{\mu}
$$

the quark one-loop collinear terms $\gamma_{C}^{i}$ read

$$
\gamma_{C}^{i}=\left[\frac{3 \alpha_{2}}{4 \pi} \eta_{i}+\frac{\alpha_{1}}{\pi} Y_{i}^{2}\right] L_{i}-\frac{9 \alpha_{2}}{16 \pi} \eta_{i}-\frac{3 \alpha_{1}}{4 \pi} Y_{i}^{2},
$$

while the Higgs one-loop collinear term $\gamma_{C}^{h}$ is

$$
\gamma_{C}^{h}=\left[\frac{3 \alpha_{2}}{4 \pi}+\frac{\alpha_{1}}{4 \pi}\right] L_{h}-\frac{3 \alpha_{2}}{4 \pi}-\frac{\alpha_{1}}{4 \pi}+\frac{3 y_{t}^{2}}{16 \pi^{2}},
$$


where we included the contribution from the top Yukawa $y_{t}$ to the Higgs wave function renormalization.

The second term $\gamma_{S}$ in Eq.(22) is the soft anomalous dimension that is obtained by summing over the soft functions [22, 29], and is reported in Ref. [24] in some detail. This soft term does not contain any large logarithm and does not contribute to the cusp anomalous dimension. It only depends on the scattering angles of the external particles, and its effect largely cancels in the integration of the cross section. At LL order this term can be neglected.

The third term $\hat{\gamma}$ in Eq. (22) is a specific new contribution occurring in the $\mathrm{VBF}$ process [23], and can be written as the sum of $S U(2)$ and $U(1)$ parts plus a term $\hat{\gamma}^{\lambda}$ arising from the rescattering of the Higgs boson. The first two parts contain cusp diagonal terms proportional to $\log \mu$, and smaller non-cusp off-diagonal terms that do not depend on the scale $\mu$. At LL order we only need to retain the diagonal cusp terms and the rescattering term that is non-cusp but is large because of the large self-coupling $\lambda$ of the Higgs boson. We also include the diagonal wave function renormalization terms. According to Ref. [24], the cusp parts of the $S U(2)$ contribution read

$$
\hat{\gamma}_{1 A, 1 B}^{S U(2)}=-\frac{3 \alpha_{2}}{8 \pi}\left[2 L_{h}+1\right]
$$

for the coefficients $C_{1 A}, C_{1 B}$,

$$
\hat{\gamma}_{4 A}^{S U(2)}=\frac{\alpha_{2}}{8 \pi}\left[2 L_{h}-4 \eta_{1}\left(L_{1}+L_{3}\right)+1\right]
$$

for the coefficient $C_{4 A}$ and

$$
\hat{\gamma}_{4 B}^{S U(2)}=\frac{\alpha_{2}}{8 \pi}\left[2 L_{h}-4 \eta_{2}\left(L_{2}+L_{4}\right)+1\right]
$$

for the coefficient $C_{4 B}$. The cusp part of the $U(1)$ contribution is

$$
\hat{\gamma}^{U(1)}=-\frac{\alpha_{1}}{8 \pi}\left[2 L_{h}+1\right],
$$

and the Higgs rescattering contribution is given by

$$
\hat{\gamma}^{\lambda}=\frac{c^{\mathcal{O}} \lambda}{4 \pi^{2}}
$$

where $c^{\mathcal{O}}=\{3,3,1,1\}$ for the coefficient $\left\{C_{1 A}, C_{1 B}, C_{4 A}, C_{4 B}\right\}$.

Summing all the terms, we find that the dependence on $L_{h}$ cancels (up to non-cusp terms like the difference $L_{h}-L_{i}$ that can be neglected at LL order), yielding the following simple result for the total anomalous dimension

$$
\gamma=\frac{3 \alpha_{2}}{4 \pi} \sum_{i} \eta_{i} L_{i}+\frac{\alpha_{1}}{\pi} \sum_{i} Y_{i}^{2} L_{i}+\gamma_{n c}
$$

where the sum is over the four external quarks. Here $\gamma_{n c}$ is the sum of the retained diagonal non-cusp terms, including constant wave function renormalization and Higgs rescattering terms

$$
\gamma_{n c}=-\frac{9 \alpha_{2}}{16 \pi}\left[\sum_{i} \eta_{i}+d^{\mathcal{O}}\right]-\frac{3 \alpha_{1}}{4 \pi}\left[\sum_{i} Y_{i}^{2}+\frac{1}{2}\right]+\frac{3 y_{t}^{2}}{16 \pi^{2}}+\hat{\gamma}^{\lambda}
$$

where $d^{\mathcal{O}}=\{2,2,10 / 9,10 / 9\}$ for the coefficient $\left\{C_{1 A}, C_{1 B}, C_{4 A}, C_{4 B}\right\}$. Some of these terms are small, but they are constant, do not depend on scattering angles, and their weight might sum up in the integration of the cross section. All other non-cusp terms have been neglected.

The simple result of Eq. (32) says that the one-loop cusp anomalous dimension is the same that we would obtain by the sum of the collinear terms for the external quarks, neglecting the Higgs particle. However the Higgs momentum would affect the kinematic of the quarks anyway. Moreover the Higgs rescattering and wave function renormalization terms are not small and have been included in the non-cusp part $\gamma_{n c}$ of the anomalous dimension.

\section{RADIATIVE CORRECTIONS AT LL ORDER}

At LL order the running of the coupling constant can be evaluated by uncoupled one-loop beta functions. That, together with the simple diagonal form of the oneloop cusp anomalous dimension in Eq.(32), allows for a fully analytical evaluation of the electroweak radiative corrections.

By insertion of Eq.(19) in Eq.(1), the low scale Wilson coefficients follow from Eq. (6) that now reads

$$
\hat{C}_{Y}\left(\mu_{l}\right)=v \sum_{Y} R_{Y X}^{(0)}\left\{\prod_{(\alpha)} \mathcal{A}_{X}^{(\alpha)}\left(\mu_{l}, \mu_{h}\right)\right\} C_{X}\left(\mu_{h}\right)
$$

where the product runs over the four couplings $\alpha=$ $\alpha_{1}, \alpha_{2}, \alpha_{y}, \alpha_{\lambda}$, having defined $\alpha_{y}=y_{t}^{2} /(4 \pi)$ and $\alpha_{\lambda}=$ $\lambda /(4 \pi)$, and the exponentiated running factors follow by integration of the corresponding anomalous dimension

$$
\mathcal{A}_{X}^{(\alpha)}\left(\mu_{l}, \mu_{h}\right)=\exp \left\{-\int_{\mu_{l}}^{\mu_{h}} \frac{\gamma_{X}^{(\alpha)}(\mu)}{\mu} \mathrm{d} \mu\right\}
$$

Here $\gamma_{X}^{(\alpha)}$ is the term proportional to the coupling $\alpha$ in the anomalous dimension for the Wilson coefficient $C_{X}$. By inspection of Eq.(32), we see that such terms can be written as functions of $t=\log \mu$

$$
\gamma_{X}^{(\alpha)}=\alpha\left[a_{\alpha} t+\mathcal{F}_{X}^{(\alpha)}(p)\right]
$$

where for each coupling $\alpha$ there is a different coefficient $a_{\alpha}$, while $\mathcal{F}_{X}^{(\alpha)}(p)$ is a function of the external momenta $p \equiv\left(p_{1}, p_{2}, p_{3}, p_{4}\right)$ that in general also depends on the chosen coefficient $C_{X}$. The explicit expressions of the 
functions are

$$
\begin{aligned}
& \mathcal{F}_{X}^{(1)}(p)=\frac{1}{\pi} \sum_{i} Y_{i}^{2} \log \left(\bar{n}_{i} \cdot p_{i}\right)-\frac{3}{4 \pi}\left[\sum_{i} Y_{i}^{2}+\frac{1}{2}\right] \\
& \mathcal{F}_{X}^{(2)}(p)=\frac{3}{4 \pi} \sum_{i} \eta_{i} \log \left(\bar{n}_{i} \cdot p_{i}\right)-\frac{9}{16 \pi}\left[\sum_{i} \eta_{i}+d^{\mathcal{O}}\right] \\
& \mathcal{F}_{X}^{(y)}(p)=\frac{3}{4 \pi} \\
& \mathcal{F}_{X}^{(\lambda)}(p)=\frac{c^{\mathcal{O}}}{\pi}
\end{aligned}
$$

while the coefficients are

$$
\begin{aligned}
& a_{1}=-\frac{1}{\pi} \sum_{i} Y_{i}^{2}, \\
& a_{2}=-\frac{3}{4 \pi} \sum_{i} \eta_{i}, \\
& a_{\lambda}=0, \\
& a_{y}=0 .
\end{aligned}
$$

In all these definitions the sums are over the external quarks. The couplings also have an implicit dependence on $t$, dictated by the one-loop beta-functions

$$
\frac{\mathrm{d} \alpha_{i}}{\mathrm{~d} t}=b_{i} \alpha_{i}^{2}
$$

These can be easily integrated, yielding

$$
\left(\frac{\alpha_{i}\left(t_{l}\right)}{\alpha_{i}\left(t_{h}\right)}\right)=1-b_{i} \alpha_{i}\left(t_{l}\right)\left(t_{h}-t_{l}\right)
$$

where the one-loop coefficients are well known 30$]$

$$
\begin{aligned}
& b_{1}=\frac{41}{12 \pi}, \\
& b_{2}=-\frac{19}{12 \pi}, \\
& b_{\lambda}=\frac{3}{\pi} \\
& b_{y}=\frac{9}{4 \pi} .
\end{aligned}
$$

By a simple integration, the running factors in Eq. 355 take the explicit general form

$$
\begin{aligned}
& \mathcal{A}_{X}^{(\alpha)}\left(\mu_{l}, \mu_{h}\right)=\left(\frac{\mu_{h}}{\mu_{l}}\right)^{\frac{a_{\alpha}}{b_{\alpha}}} \\
& \quad \times\left(\frac{\alpha\left(t_{l}\right)}{\alpha\left(t_{h}\right)}\right)\left[\frac{1}{b_{\alpha}}\left(\frac{a_{\alpha}}{b_{\alpha} \alpha\left(t_{l}\right)}+a_{\alpha} t_{l}+\mathcal{F}_{X}^{(\alpha)}(p)\right)\right]
\end{aligned}
$$

and their product in Eq.(34) gives an analytical expression for the electroweak radiative correction at LL order. The cross section follows by insertion of the low scale Wilson coefficients from Eq. 34 in the phase space integral of Eq.(5) and integrating by PDFs. The closed analytical form of Eq.42) greatly speeds up the numerical integration of the cross section at LL order compared with NLL order. We discuss the accuracy of the two orders in the next section by a direct comparison.

\section{LL VS. NLL ORDER: NUMERICAL RESULTS}

In this section the accuracy of the approximation is tested by a comparison between LL and NLL orders. All the details of the numerical integration are kept exactly the same as reported in Ref. [24] where the cross section was evaluated at NLL order. We summarize them briefly. The low energy matching scale is chosen to be $\mu_{l}=M_{Z}$. The couplings and parameters of the standard model have been set at the electroweak scale $\mu_{l}=M_{Z}$ according to the data of Ref.[31]. The Higgs mass is assumed to be $M_{H}=125 \mathrm{GeV}$. The high scale $\mu_{h}$ is set at the larger value between $M_{Z}$ and the geometric average $\mu^{2}=\sqrt{t_{3} t_{4}}$. At this scale the sum of logarithmic terms $\ln \left(-t_{3} / \mu^{2}\right)+\ln \left(-t_{4} / \mu^{2}\right)$ reaches its minimum in the oneloop matching. As discussed in Ref. [24] this choice has the merit of stopping the running whenever one of the Mandelstam variables is too small, while keeping the neglected one-loop matching terms as small as possible. On the other hand the sensitivity to the choice of the high scale was shown to be small and comparable to the sensitivity of the standard tree-level cross section. ${ }^{1} \mathrm{We}$ use CTEQ6 PDFs [33] and neglect the very small contribution of $t$ and $b$ quarks. Moreover we neglect the s-channel contribution and interference terms that are known to be small with VBF cuts. The masses of the vector gauge bosons are restored in the denominators of the coefficients in Eq. (14), as their effects become important when the total cross section is evaluated. Hereafter, in order to compare with the NLL calculation of Ref. 24], we adopt the same cuts on angles and transverse moment: $\theta_{3}>10^{\circ}, \theta_{4}<170^{\circ}, p_{T}>20 \mathrm{GeV}$. No QCD corrections have been included in both calculations, and only the virtual electroweak corrections are considered by the method.

The terms contributing to the cross section at tree-level are reported in Fig.2. The $u d \rightarrow d u$ process dominates, followed by the other Left-Left terms. The Left-Right contributions are two order of magnitude smaller, while the Right-Right terms are very small and have been neglected. In Fig. 2 a sum over the generations of quarks is included through the PDFs.

As a first comparison we study the single terms in the phase space, and for each of them we calculate the K-

\footnotetext{
1 At variance with variational approaches the principle of minimal sensitivity does not hold. The best choice of $\mu_{h}$ should make the omitted terms small, as for the method of minimal variance 32 .
} 


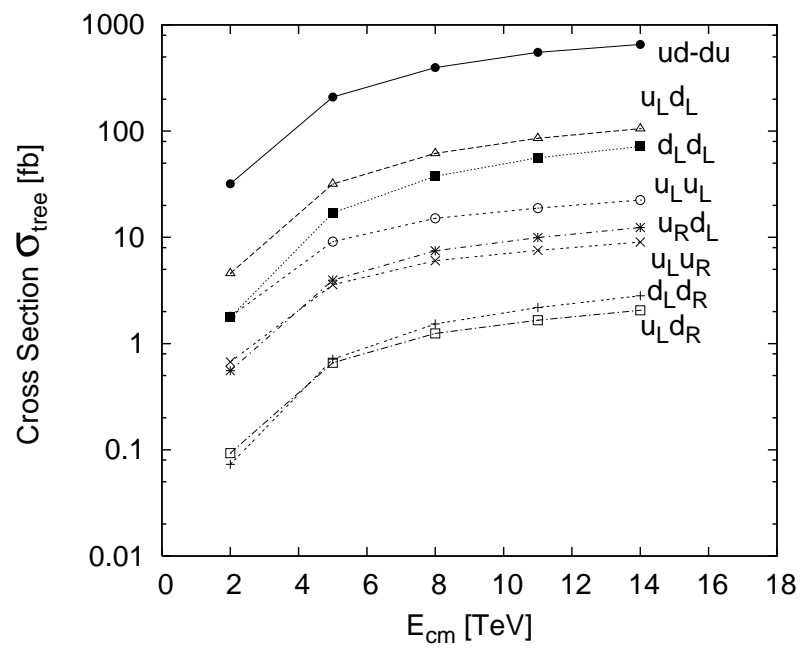

FIG. 2: Terms contributing to the cross section at tree-level, as a function of $E_{\mathrm{cm}}$, with the cuts: $\theta_{3}>10^{\circ}, \theta_{4}<170^{\circ}$, $p_{T}>20 \mathrm{GeV}$.

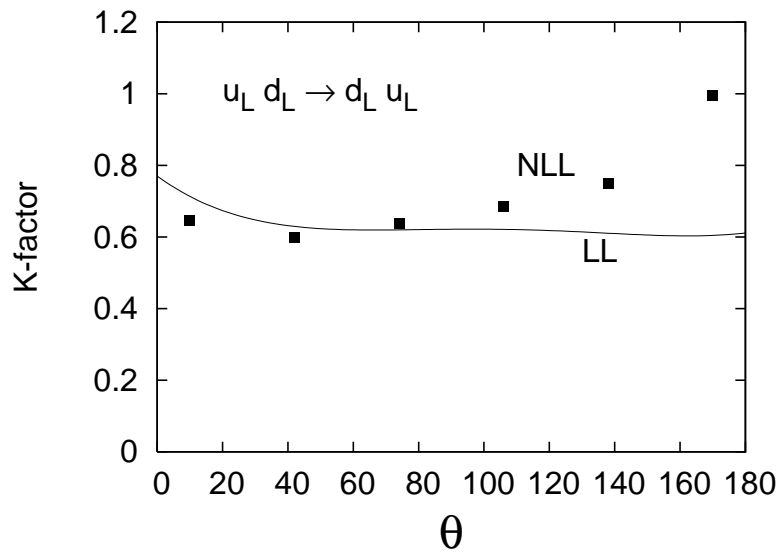

FIG. 3: The factor $K=\sigma_{E W} / \sigma_{\text {tree }}$ for the process $u_{L} d_{L} \rightarrow$ $d_{L} u_{L}$ as a function of $\theta=\theta_{3}=180^{\circ}-\theta_{4}$, at $\varphi=90^{\circ}, x_{1}=$ $x_{2}=0.5, E_{\mathrm{cm}}=14 \mathrm{TeV}$ and $E_{3}=x_{1} x_{2} E_{\mathrm{cm}} / 2$. The result at LL order (solid line) is compared with the NLL order data points (squares).

factor which is defined as the ratio between the cross section with electroweak corrections on, and the tree-level cross section without any radiative correction

$$
K=\frac{\sigma_{E W}\left(\theta_{3}, \theta_{4}, \varphi\right)}{\sigma_{\text {tree }}\left(\theta_{3}, \theta_{4}, \varphi\right)}
$$

where the differential cross sections are evaluated for fixed values of $x_{1}, x_{2}, E_{3}$ and $E_{\mathrm{cm}}$, and for a given set of angles. Here the simple LL calculation is compared with the NLL result of Ref. 24]. In Figs. 3-10 the Kfactor is reported as a function of $\theta=\theta_{3}$ with $\theta_{4}=\pi-\theta_{3}$ and $\varphi=\pi / 2$ at a typical set of parameters: $E_{\mathrm{cm}}=14$ $\mathrm{TeV}, x_{1}=x_{2}=0.5$ and $E_{3}=x_{1} x_{2} E_{\mathrm{cm}} / 2$. The agreement of LL and NLL results is very good at small angles. As expected, at LL order the dependence on angles is

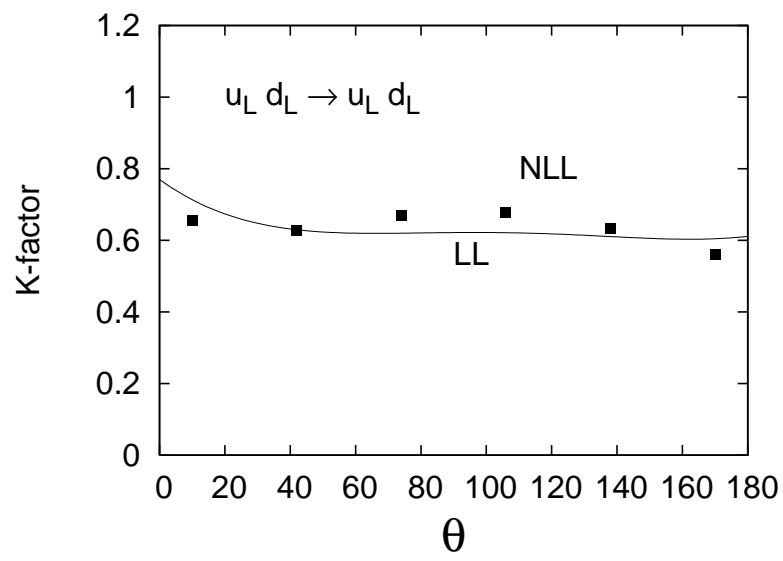

FIG. 4: The same as Fig.3 for the process $u_{L} d_{L} \rightarrow u_{L} d_{L}$.

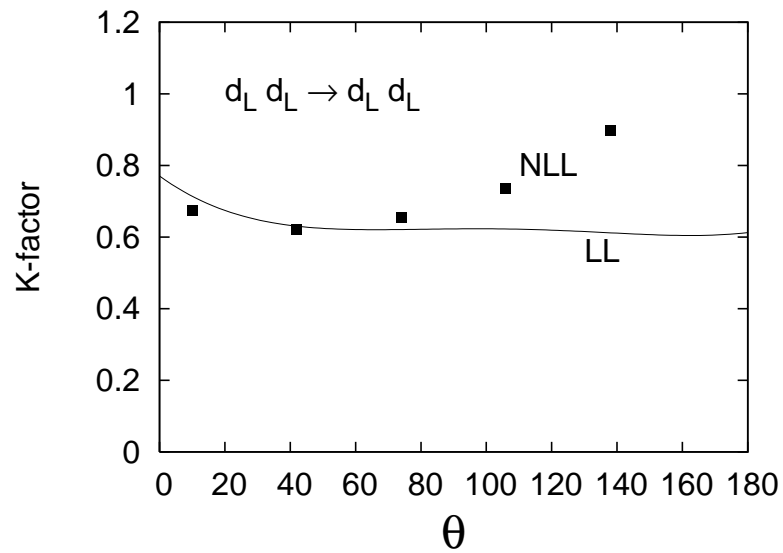

FIG. 5: The same as Fig.3 for the process $d_{L} d_{L} \rightarrow d_{L} d_{L}$.

reduced in comparison with the NLL result, because the neglected terms have a larger dependence on the scattering angles. However for $\theta<90^{\circ}$, in the physically relevant phase-space region, the difference is small and the LL result (solid line) interpolate the NLL data (squares)

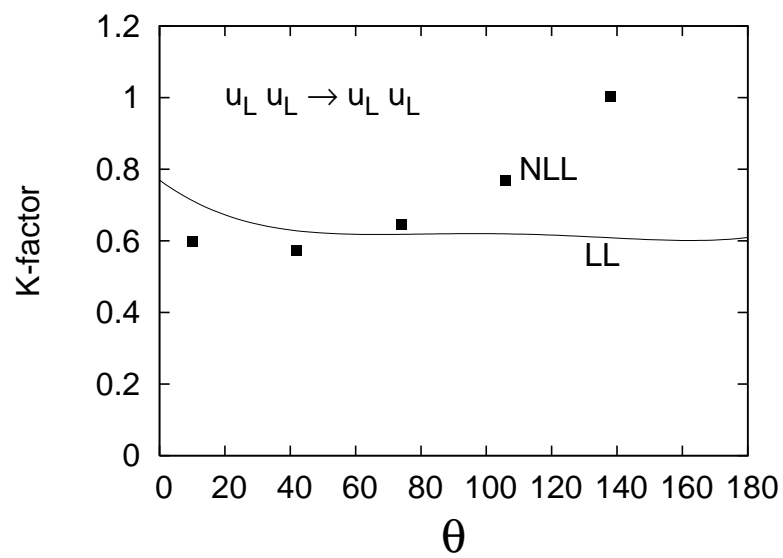

FIG. 6: The same as Fig.3 for the process $u_{L} u_{L} \rightarrow u_{L} u_{L}$. 


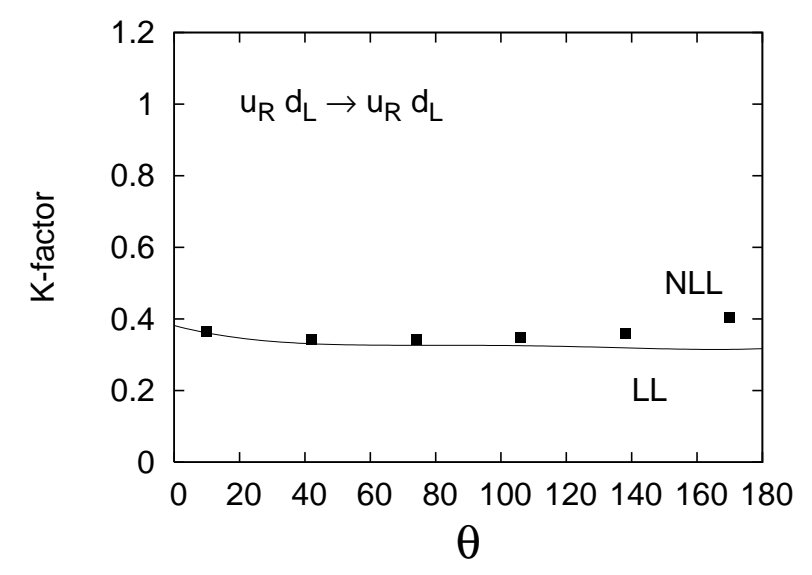

FIG. 7: The same as Fig.3 for the process $u_{R} d_{L} \rightarrow u_{R} d_{L}$.

quite well. The dependence on the azimuthal angle $\phi$ is very small and negligible for $\theta<90^{\circ}$, in perfect agreement with the NLL order.

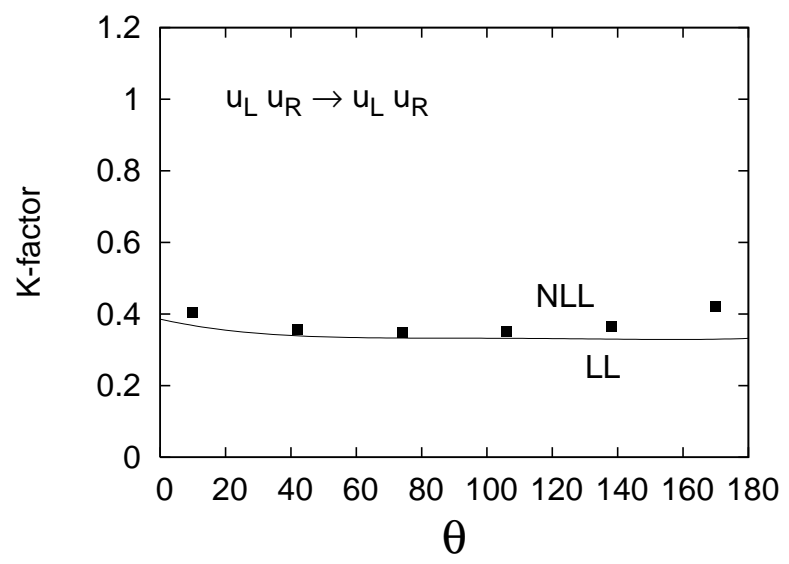

FIG. 8: The same as Fig.3 for the process $u_{L} u_{R} \rightarrow u_{L} u_{R}$.

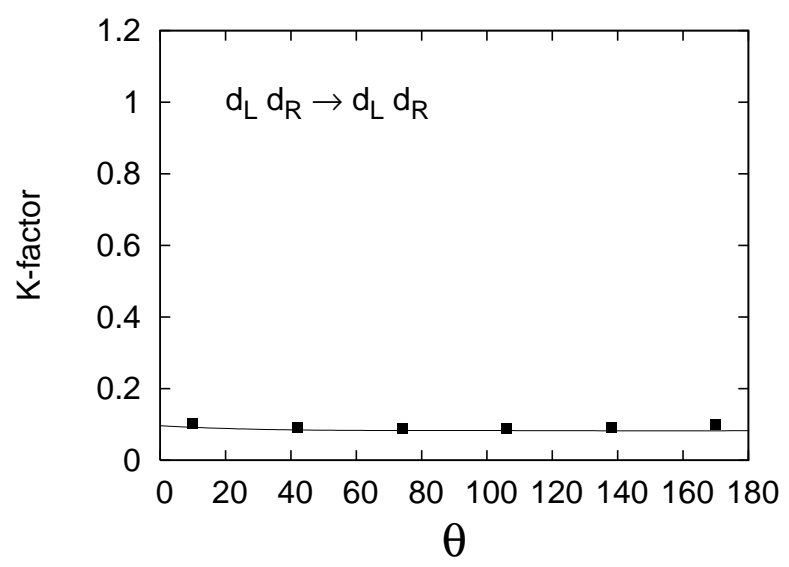

FIG. 9: The same as Fig.3 for the process $d_{L} d_{R} \rightarrow d_{L} d_{R}$.
We find a better agreement for the Left-Right processes (Fig. 7-10) where the radiative corrections are larger,

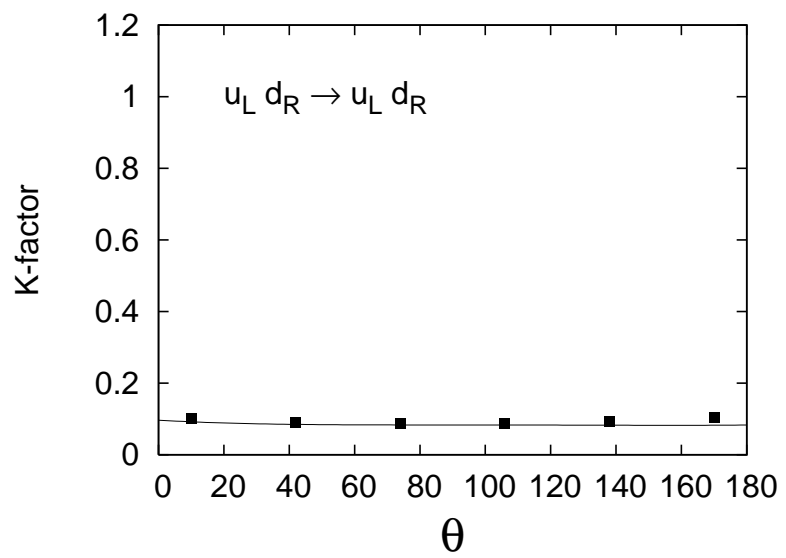

FIG. 10: The same as Fig.3 for the process $u_{L} d_{R} \rightarrow u_{L} d_{R}$.

than for Left-Left processes (Fig. 3-6) where the corrections are smaller. Thus the very large suppression of the Left-Right processes is mainly due to the role played by the collinear anomalous dimension. It is remarkable that such large suppression has no relevant effects on the total cross section which is dominated by the Left-Left processes.

The single terms are integrated by PDFs over the phase space and summed up, yielding the cross section $\sigma_{E W}$. By comparison with the tree level cross section, the relative electroweak correction is defined as the ratio

$$
\frac{\Delta \sigma}{\sigma}=\frac{\sigma_{E W}-\sigma_{\text {tree }}}{\sigma_{\text {tree }}}
$$

and is displayed in Fig.11 as a function of the center-ofmass energy. For comparison the NLL result of Ref. 24] is also reported in Fig.11 together with the output of the code HAWK [5, 6] that is based on a fixed order perturbative calculation.

While differences are negligible below $2 \mathrm{TeV}$, at large energies LL and NLL corrections grow faster than predicted by fixed order one-loop calculations, reaching $9 \%$ at the full LHC energy $E_{\mathrm{cm}}=14 \mathrm{TeV}$, to be compared with $5 \%$ predicted by HAWK. At LL order the correction is a bit smaller with respect to NLL order, but the difference is less than $1 \%$ up to $E_{\mathrm{cm}}=10 \mathrm{TeV}$. Thus the simple LL calculation provides an analytical electroweak correction that seems to be more reliable than fixed order calculations at high energies. While fixed-order perturbative calculations might miss part of the correction at the LHC energy scale, the simple LL resummation contains the main terms, and gives integrated corrections that are more accurate than PDFs. Insertion of the simple analytical result of Eqs.(34), (42) in software packages would be straightforward, and would increase the accuracy of simulations. 


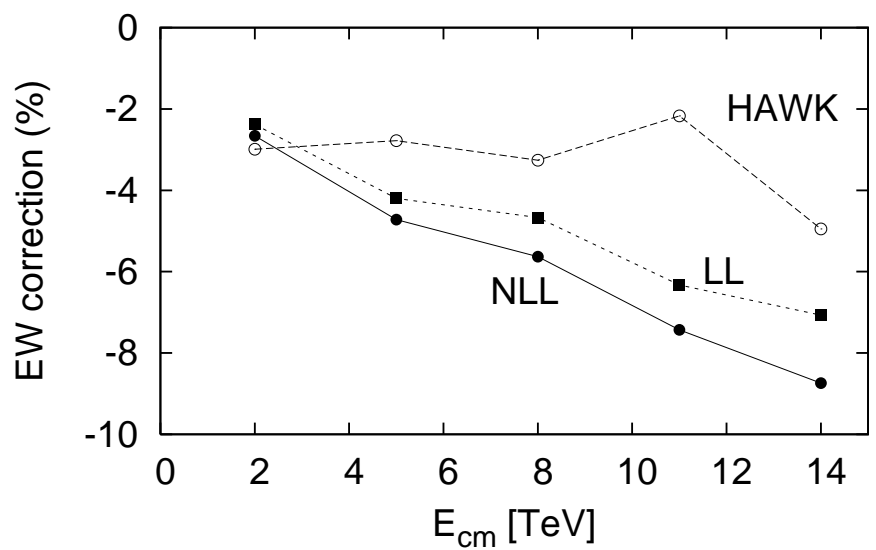

FIG. 11: Relative electroweak correction $\left[\sigma_{E W}-\right.$ $\left.\sigma_{T R E E}\right] / \sigma_{\text {tree }}$ as a function of the center-of-mass energy $E_{\mathrm{cm}}$ evaluated by Eq. (5) at LL order (filled squares), compared with the NLL order of Ref. 24] (filled circles) and with the output of HAWK (open circles). Cuts are: $p_{T}>20$ $\mathrm{GeV}, \theta_{3}>10^{\circ}, \theta_{4}<170^{\circ}$.

[1] S. Chatrchyan et al. [The CMS Collaboration], Phys. Lett. B 716, 30 (2012), arXiv:1207.7235v2.

[2] G. Aad et al. [The ATLAS Collaboration], Phys. Lett. B 716, 1 (2012), arXiv:1207.7214v2.

[3] M. Duhrssen, S. Heinemeyer, H. Logan, D. Rainwater, G. Weiglein, et al., Phys.Rev. D70, 113009 (2004), hep$\mathrm{ph} / 0406323$.

[4] D. Zeppenfeld, R. Kinnunen, A. Nikitenko, and E. Richter-Was, Phys.Rev. D62, 013009 (2000), hepph/0002036.

[5] M. Ciccolini, A. Denner, and S. Dittmaier, Phys. Rev. Lett. 99, 161803 (2007), arXiv:0707.0381.

[6] M. Ciccolini, A. Denner, and S. Dittmaier, Phys. Rev. D77, 013002 (2008), arXiv:0710.4749.

[7] T. Han, G. Valencia, and S. Willenbrock, Phys.Rev.Lett. 69, 3274 (1992), hep-ph/9206246.

[8] T. Figy, D. Zeppenfeld, and C. Oleari, Phys. Rev. D68, 073005 (2003), hep-ph/0306109.

[9] E. L. Berger and J. M. Campbell, Phys.Rev. D70, 073011 (2004), hep-ph/0403194.

[10] K. Arnold, M. Bahr, G. Bozzi, F. Campanario, C. Englert, et al., Comput.Phys.Commun. 180, 1661 (2009), arXiv:0811.4559.

[11] R. V. Harlander, J. Vollinga, and M. M. Weber, Phys.Rev. D77, 053010 (2008), arXiv:0801.3355.

[12] P. Bolzoni, F. Maltoni, S.-O. Moch, and M. Zaro, Phys. Rev. Lett. 105, 011801 (2010), arXiv:1003.4451.

[13] P. Bolzoni, M. Zaro, F. Maltoni, and S.-O. Moch, Nucl. Phys. Proc. Suppl. 205-206, 314 (2010), arXiv:1006.2323.

[14] P. Bolzoni, F. Maltoni, S.-O. Moch, and M. Zaro, Phys.Rev. D85, 035002 (2012), arXiv:1109.3717.

[15] C. W. Bauer, S. Fleming, and M. E. Luke, Phys. Rev. D63, 014006 (2000), hep-ph/0005275.

[16] C. W. Bauer, S. Fleming, D. Pirjol, and I. W. Stewart, Phys. Rev. D63, 114020 (2001), hep-ph/0011336.

[17] C. W. Bauer and I. W. Stewart, Phys. Lett. B516, 134
(2001), hep-ph/0107001.

[18] C. W. Bauer, D. Pirjol, and I. W. Stewart, Phys. Rev. D65, 054022 (2002), hep-ph/0109045.

[19] J.-y. Chiu, F. Golf, R. Kelley, and A. V. Manohar, Phys. Rev. D77, 053004 (2008), arXiv:0712.0396.

[20] J.-y. Chiu, F. Golf, R. Kelley, and A. V. Manohar, Phys. Rev. Lett. 100, 021802 (2008), arXiv:0709.2377.

[21] J.-y. Chiu, R. Kelley, and A. V. Manohar, Phys. Rev. D78, 073006 (2008), arXiv:0806.1240.

[22] J.-y. Chiu, A. Fuhrer, R. Kelley, and A. V. Manohar, Phys. Rev. D80, 094013 (2009), arXiv:0909.0012.

[23] A. Fuhrer, A. V. Manohar, and W. J. Waalewijn, Phys.Rev. D84, 013007 (2011), arXiv:1011.1505.

[24] F. Siringo and G. Buccheri, Phys. Rev. D86, 053013 (2012), arXiv:1207.1906.

[25] R. N. Cahn, S. D. Ellis, R. Kleiss, and W. J. Stirling, Phys.Rev. D35, 1626 (1987).

[26] V. D. Barger, R. Phillips, and D. Zeppenfeld, Phys.Lett. B346, 106 (1995), hep-ph/9412276.

[27] V. Del Duca, G. Klamke, D. Zeppenfeld, M. L. Mangano, M. Moretti, et al., JHEP 0610, 016 (2006), hep$\mathrm{ph} / 0608158$.

[28] F. Siringo, Eur. Phys. J. C32, 555 (2004), arXiv:hep$\mathrm{ph} / 0307320 \mathrm{v} 1$.

[29] J.-y. Chiu, A. Fuhrer, R. Kelley, and A. V. Manohar, Phys. Rev. D81, 014023 (2010), arXiv:0909.0947.

[30] H. Arason, D. J. Castano, B. Kesthelyi, S. Mikaelian, E. J. Piard, P. Ramond, and B. D. Wright, Phys. Rev. D 46, 3945 (1992).

[31] S. Eidelman et al. [Particle Data Group], Phys. Lett. B592, 1 (2004).

[32] F. Siringo and L. Marotta, Eur. Phys. J. C44, 293 (2005), arXiv:hep-ph/0506284.

[33] J. Pumplin, A. Belyaev, J. Huston, D. Stump, and W. K. Tung, JHEP 0602, 032 (2006), hep-ph/0512167. 